\title{
The effect of laser wavelength on the ablation rate of carbon
}

\author{
J. Hoffman · J. Chrzanowska $\cdot$ S. Kucharski $\cdot$ \\ T. Moscicki - I. N. Mihailescu $\cdot$ C. Ristoscu • \\ Z. Szymanski
}

Received: 4 November 2013/ Accepted: 12 May 2014/Published online: 7 June 2014

(C) The Author(s) 2014. This article is published with open access at Springerlink.com

\begin{abstract}
The ablation of graphite is studied as a function of laser fluence for 355, 532 and 1,064 nm wavelength generated by a nanosecond Nd:YAG laser. It has been found that in the case of lower wavelengths, the transition from the thermal ablation to the phase explosion takes place at lower laser fluences. The change of crater shape due to the effect of deep drilling in the proximity of the phase explosion threshold was observed. The calculations of plasma radiation flux to the target surface were made, and the considerable increase of absorbed energy density was found in the case of $355 \mathrm{~nm}$ wavelength.
\end{abstract}

\section{Introduction}

Pulsed laser ablation is a well-established method of removing material from a solid surface and synthesizing nanostructures. In most of its technological applications, the ablation threshold, the ablation rate and transition from the thermal ablation to the phase explosion are key parameters. The last effect-sudden increase of the laser ablation rate over certain laser fluence-was observed and studied in several papers [1-7]. It is rather general agreement that this increase corresponds to a change of the ablation mechanism from surface vaporization to explosive boiling (also known as phase explosion). It is caused by

J. Hoffman $(\bowtie) \cdot$ J. Chrzanowska $\cdot$ S. Kucharski .

T. Moscicki · Z. Szymanski

Institute of Fundamental Technological Research, Pawinskiego

5B, 02-106 Warsaw, Poland

e-mail: jhoffman@ippt.gov.pl

I. N. Mihailescu $\cdot$ C. Ristoscu

National Institute for Lasers, Plasma and Radiation Physics,

Atomistilor 409, 77125 Bucharest, Romania rapid target heating and, therefore, is a function of the laser pulse energy per unit area (fluence). Rapid laser heating causes that the heated liquid-vapor system leaves the saturation line and enters the metastable region. According to the theory of superheated liquids when a liquid reaches its homogeneous nucleation temperature $T_{\mathrm{hn}} \geq 0.9 T_{\mathrm{cr}}$ (where $T_{\text {cr }}$ denotes critical temperature), rapid transition from superheated liquid to a mixture of vapor and liquid occurs. Since the volume of gas phase greatly exceeds that of liquid, sudden boiling resembles an explosion [8] and results in ejection of mixture of vapor and liquid droplets.

The transition to phase explosion was visualized in [5], and the images showed that the effect takes place after termination of the laser pulse due to the time lag for formation of homogeneous nuclei. Another explanation of this delay suggested by Tao et al. [7] is that it corresponds to the time after the cessation of the laser pulse, which is necessary to decrease the recoil pressure exerted upon the surface of the target melt pool by the vaporization flux and plasma pressure. Since the ejection of droplets results also in very deep craters, the phenomenon is important from a practical point of view because of application of laser ablation in many areas of technology such as laser surgery, laser cleaning, surface patterning, deposition of thin films or synthesis of nanostructures. For example, the production of large particulates as well as nanoparticles that appear as a result of phase explosion seriously diminishes the quality of the deposited thin films.

In this paper, the ablation of graphite is studied as a function of laser fluence for various wavelength of a Nd:YAG laser. The ablation of graphite is used to obtain a wide variety of carbon allotropes, such as diamond-like carbon films [9, 10], diamond crystallites [11] fullerene carbon molecules [12], carbon nanotubes [13, 14], carbon nanowalls [15] and graphene [16, 17] as well as for the 
removal of co-deposited layers from plasma limiters in tokamaks [18]. However, there are only a sparse measurements of such factors as the ablation or explosive boiling threshold for nanosecond laser pulse [3, 4, 19-21]. In this work, the results of systematic measurements of the ablation rate for the first, second and third harmonic of $\mathrm{Nd}$ :YAG laser in wide range of laser fluences are presented. The crater cross sections are also shown. Both polycrystalline graphite and highly oriented pyrolytic graphite were used in experiments. The results of theoretical calculations are also presented and compared with the experiment.

\section{Experiment}

Irradiation of a graphite target was performed in a chamber evacuated to a residual pressure of $1 \times 10^{-5} \mathrm{~Pa}$ using a Nd:YAG laser (Quantel, $981 \mathrm{E}$ ). The laser was operated at a wavelength of $1,064,532$ or $355 \mathrm{~nm}$ with $10 \mathrm{~ns}$ pulse duration (FWHM) and a repetition rate of $10 \mathrm{~Hz}$. All harmonics are polarized horizontally. The spatial and time profiles of the laser beam were Gaussian. The laser fluence was changed from 1 to $150 \mathrm{~J} \mathrm{~cm}^{-2}$. The laser beam spot size at the target surface was kept constant while the laser pulse energy was varied. The laser spot diameter defined by $I_{0} / e^{2}$, where $I_{0}$ is peak intensity, was determined by registration of the focal spot with ICCD camera after attenuation of the laser beam and was $\sim 310 \mu \mathrm{m}$ in the case of 1,064 nm and $\sim 260 \mu \mathrm{m}$ in the case of 355 and $532 \mathrm{~nm}$ wavelength.

The incident angle of the laser beam was close to the surface normal. Two types of graphite-a polycrystalline graphite from Goodfellow (1.8 $\mathrm{g} \mathrm{cm}^{-3}$ mass density) and highly oriented pyrolytic graphite (HOPG) from Kurt J.Lesker $\left(2.2 \mathrm{~g} \mathrm{~cm}^{-3}\right)$-were used. The target was rotated to avoid crater formation. At each fluence, the amount of ablated substance was determined by measuring the target weight before and after many thousand shots. In the presented range of fluence $(2.5 \leq F \leq 110)$, the number of laser shots changes from 90,000 to 9,000 . Craters formed after single or several laser shots were also examined. The measurements were made with the use of scanning profilometer Homme Tester T 8000 nanoscan and optical profilometer.

\section{Results and analysis}

The results of experiments are shown in Figs. 1 and 2. The ablation rate (total ablated mass per number of pulses per laser spot area) for different laser wavelengths is shown in Fig. 1 as a function of laser fluence up to $10 \mu \mathrm{g} \mathrm{mm}^{-2}$ pulse $^{-1}$ only. The further growth of the ablation rate between 10 and $25 \mu \mathrm{g} \mathrm{mm}^{-2}$ pulse $^{-1}$ is very steep and is not shown in the figure. The transition to the phase explosion is indicated by the jump of the ablation rate. The transition takes place at approximately $F \approx 10 \mathrm{~J} \mathrm{~cm}^{-2}$ for $355 \mathrm{~nm}, F \approx 25 \mathrm{~J} \mathrm{~cm}^{-2}$ for $532 \mathrm{~nm}$ and $F \approx 55 \mathrm{~J} \mathrm{~cm}^{-2}$ for $1,064 \mathrm{~nm}$. It is worth noting that in the case of $532 \mathrm{~nm}$, the explosive vaporization threshold is observed at the same fluence as in Ref. [4]. In the case of 355 and $1,064 \mathrm{~nm}$, similar results are obtained for highly oriented pyrolytic graphite (Fig. 1b); although for $355 \mathrm{~nm}$, the transition is not seen because of too few measurements at low fluence. For $1,064 \mathrm{~nm}$, the transition is less pronounced, and for that reason, it is shown in smaller scale in the inset. The lower threshold for the transition to explosive boiling in the case of lower wavelengths is not surprising given the fact that the absorption coefficient of carbon decreases for longer wavelength. The critical point is therefore reached at lower fluences for lower wavelengths.

The lack of distinct difference in the ablation rate for polycrystalline graphite and HOPG is by no means surprising since in the liquid phase the target looses its crystalline structure. Minor differences can result from different mass density of the targets.

The cross sections of craters ablated from polycrystalline rigid foil by 1,064, 532 and $355 \mathrm{~nm}$ radiation are shown in Fig. 2. The ablated rate (obtained from the crater volume multiplied by mass density of graphite) is in fair agreement with that obtained from the weight loss. For the sake of visualization, the crater depth is shown in smaller scale than the crater width.

In the case of 1,064 $\mathrm{nm}$ wavelength, the ablated area is always essentially of the same size as the measured laser spot (Fig. 2a). However, at fluence $F \approx 50 \pm 5 \mathrm{~J} \mathrm{~cm}^{-2}$ at the bottom of the wide, shallow crater much thinner, deep channel appears. Similar change of crater shape was observed at $F \approx 17 \mathrm{~J} \mathrm{~cm}^{-2}$ in the case of $532 \mathrm{~nm}$ radiation (Fig. 2b), and at $F \approx 7.5 \mathrm{~J} \mathrm{~cm}^{-2}$ in the case of $355 \mathrm{~nm}$ radiation (Fig. 2c). This thin channel although conspicuous does not really increase the ablated mass more than 10-15\%. However, the change of the crater shape at fluence close to the phase explosion threshold indicates the change of the ablation mechanism. It is understandable that in the case of Gaussian beam, the critical temperature is reached first in the center. When fluence exceeds the explosive vaporization threshold, the craters change their shape definitely and their depth is always much higher than that resulting from the conductive heat transport. This is clearly demonstrated in the case of third harmonic when at $F \geq 20 \mathrm{~J} \mathrm{~cm}^{-2}$ craters over $20 \mu \mathrm{m}$ deep are obtained already after single laser shot (Fig. 2d), while in the range $3.3 \leq F \leq 7$, the ablation depth is about $300 \mathrm{~nm} /$ pulse. The area of ablated target surface is at low fluences smaller 
Fig. 1 Ablation rate for various laser wavelengths as function of laser fluence. a Polycrystalline graphite. b Highly oriented pyrolytic graphite
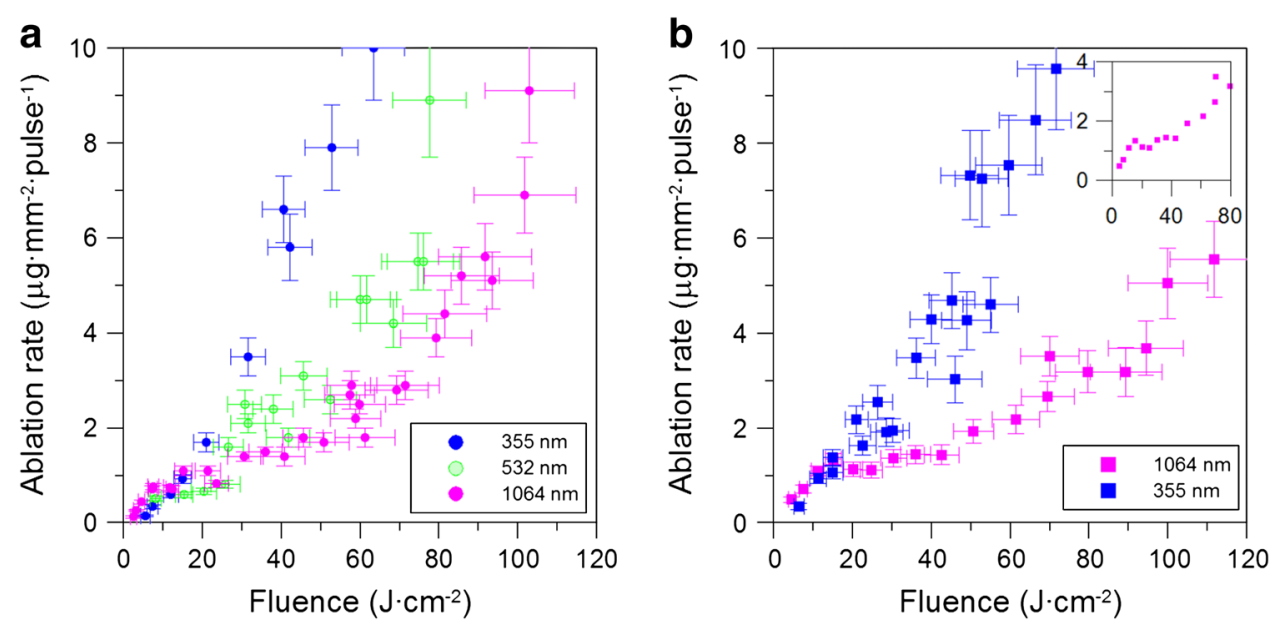

Fig. 2 Cross section of craters for various laser wavelengths and fluences. Target-

Goodfellow rigid foil. a Laser wavelength $1,064 \mathrm{~nm}, 3$ laser pulses, fluence 40,55 and $80 \mathrm{~J} \mathrm{~cm}^{-2}$. b $532 \mathrm{~nm}, 10$ laser pulses, fluence $15,17.5$ and $25 \mathrm{~J} \mathrm{~cm}^{-2}$. c $355 \mathrm{~nm}, 10$ laser pulses, fluence $3.3,7.5$ and $10 \mathrm{~J} \mathrm{~cm}^{-2}$. d $355 \mathrm{~nm}, 1$ laser pulse, fluence 20,30,60 and $100 \mathrm{~J} \mathrm{~cm}^{-2}$
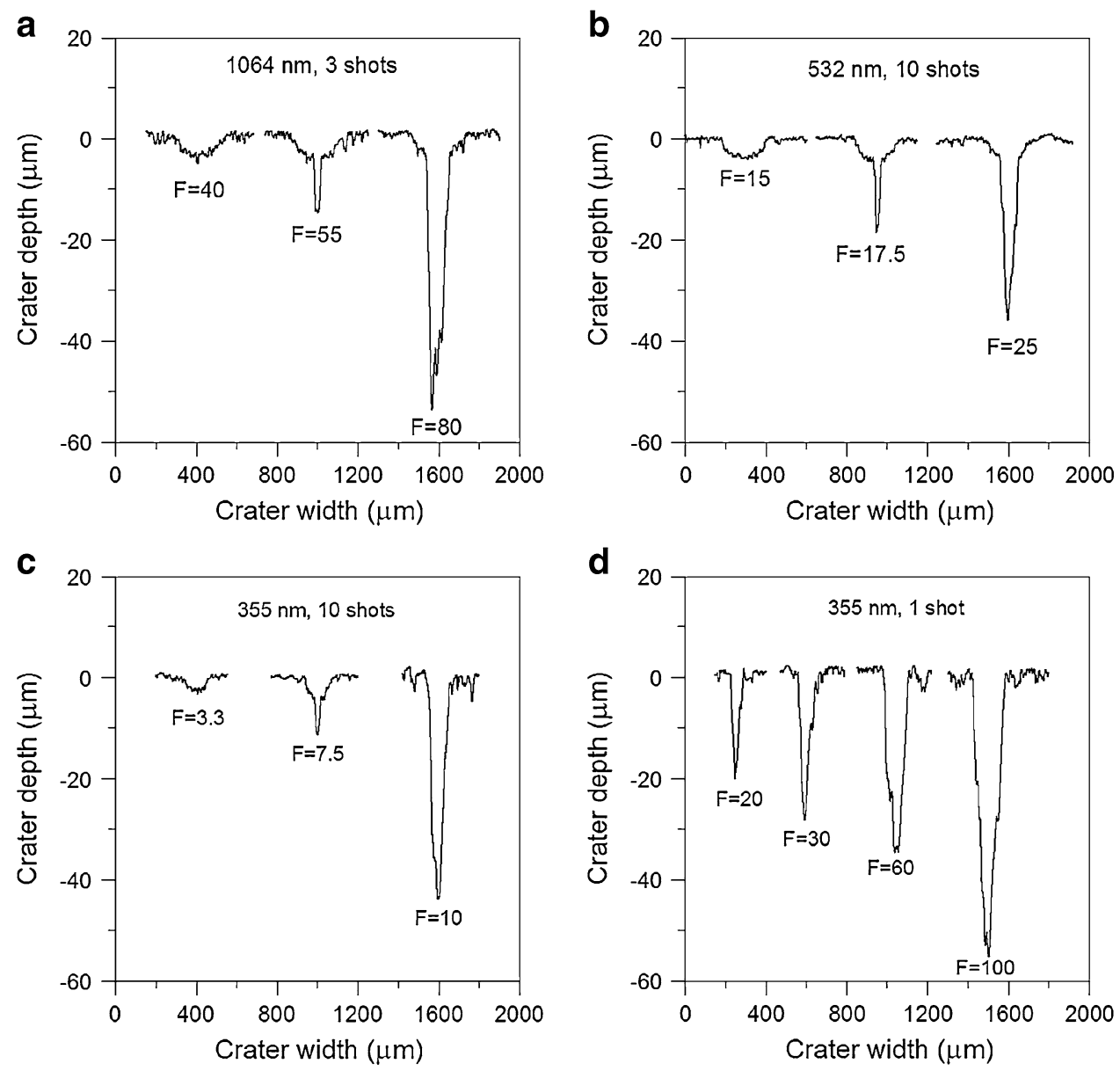

than that obtained from the laser spot measurements and gradually increases with fluence. This is well comprehensible in the case of Gaussian beam because the ablation threshold shifts to bigger beam radius with the increase of fluence. The measured diameters of the ablated area were used to construct $r^{2} \sim \ln (F)$ plot and to determine the ablation threshold, which was found about $1 \mathrm{~J} \mathrm{~cm}^{-2}$ in the case of third harmonic.

\section{Modelling}

The theoretical model that describes the target heating, formation of the plasma and its expansion, presented in [22], was used to calculate the ablation rate. The results are shown in Fig. 3. The thermal model used should properly describe the ablation process at laser fluence lower than the phase explosion threshold. The model predicts a reasonable 
crater's depth below the threshold. However, other results of calculations do not fit well the experimental findings. At low fluences $\left(F \leq 10 \mathrm{~J} \mathrm{~cm}^{-2}\right)$, the experimental ablation rate is higher for the $1,064 \mathrm{~nm}$ than for the lower wavelengths. This is contrary to the expectations because the reflectivity grows with the wavelength while the absorption coefficient decreases [23, 24]. The optical properties of graphite were used after Ref. [23] because they are very close to those found for the liquid phase of carbon in [24].

In [4], the role of radiation from the laser-induced plasma in deepening the molten layer and in consequence the crater depth during the explosive vaporization was found. Considerable difference between temperature and pressure distributions of plasma plume induced by 355 and $1,064 \mathrm{~nm}$ radiation found in [22] suggested wavelengthdependent plasma effects on the ablation rate. In this work, such distributions were consequently used to calculate

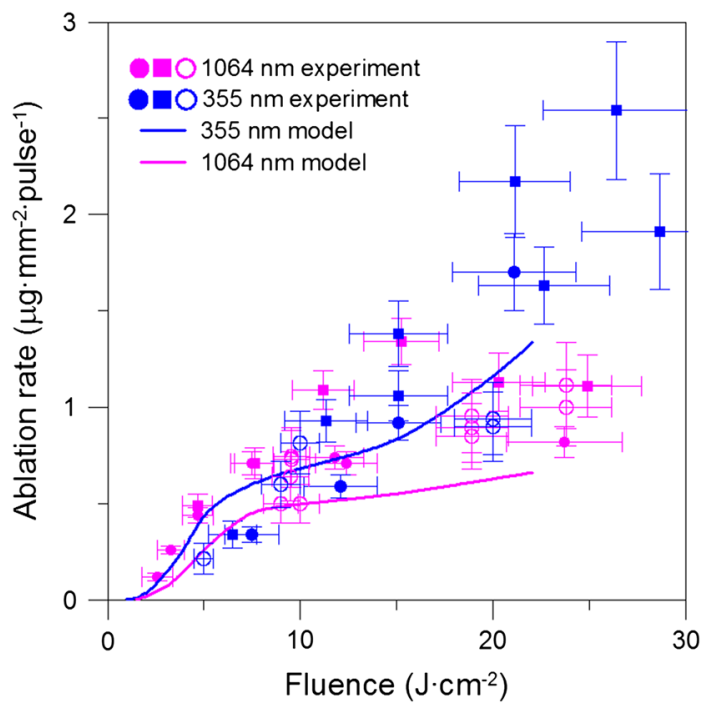

Fig. 3 Ablation rate. Lines-calculations. Symbols-experiment (filled symbols-from weight measurements, open symbols-from crater's volume) plasma composition at $F=15 \mathrm{~J} \mathrm{~cm}^{-2}$ and time interval $5 \leq t \leq 30 \mathrm{~ns}$. The beginning of the laser pulse (10 ns FWHM) was assumed $10 \mathrm{~ns}$ prior to its maximum intensity (see Fig. 5 of Ref. [22]). The characteristic distributions of the plasma temperatures and the electron densities at $t=15 \mathrm{~ns}$ are shown in Figs. 4 and 5.

Next, the radiation flux to the target surface was calculated. The calculations, similar to that presented in [4], included all possible radiation mechanisms: the electronatom and the electron-ion bremsstrahlung, the radiative recombination and line radiation. The most important term - the photo-recombination - was calculated with the use of carbon photoionization cross sections [25]. The range of wavelength was $140-1,100 \mathrm{~nm}$, and the corresponding absorption coefficients were taken from [23]. The calculations revealed that the plasma radiation increases the absorbed energy density $\sum_{\lambda} \alpha(\lambda) \cdot F(\lambda)$, where $\alpha$ is the absorption coefficient of graphite and $\lambda$ is the wavelength. Taking into account that the laser radiation is strongly absorbed by the plasma plume [22], the plasma radiation increases the absorbed energy density by 22.5 and $3.8 \%$, for 355 and $1,064 \mathrm{~nm}$, respectively. This means that plasma radiation can significantly modify the ablation process in the case of lower wavelength. It results from the fact that dominant plasma radiation is due to the photorecombination; the resulting continuum radiation is $\int_{\lambda} \varepsilon(\lambda) \mathrm{d} \lambda \approx N_{e} \cdot T^{-0.5} \sum_{z} Z^{2} \cdot N_{z}$ where $N_{e}$ and $N_{z}$ are the electron and ion density. Such denser and cooler plasma is produced by shorter laser radiation.

This effect together with the explosive vaporization can explain the formation of deep craters at fluences higher than the threshold for the phase explosion. It is rather obvious that the surface vaporization alone cannot result in deep drilling and consideration of volumetric mass removal is necessary. However, it is still not clear why at low fluence $\left(F \leq 10 \mathrm{~J} \mathrm{~cm}^{-2}\right)$ the observed ablation rate was smaller in the case of second and third harmonic than that at $1,064 \mathrm{~nm}$. It is worth noting that similar effect was found
Fig. 4 Distribution of the plasma temperature and the electron density at $15 \mathrm{~ns}$ from the beginning of the $355 \mathrm{~nm}$ laser pulse. Laser fluence $15 \mathrm{~J} \mathrm{~cm}^{-2}$
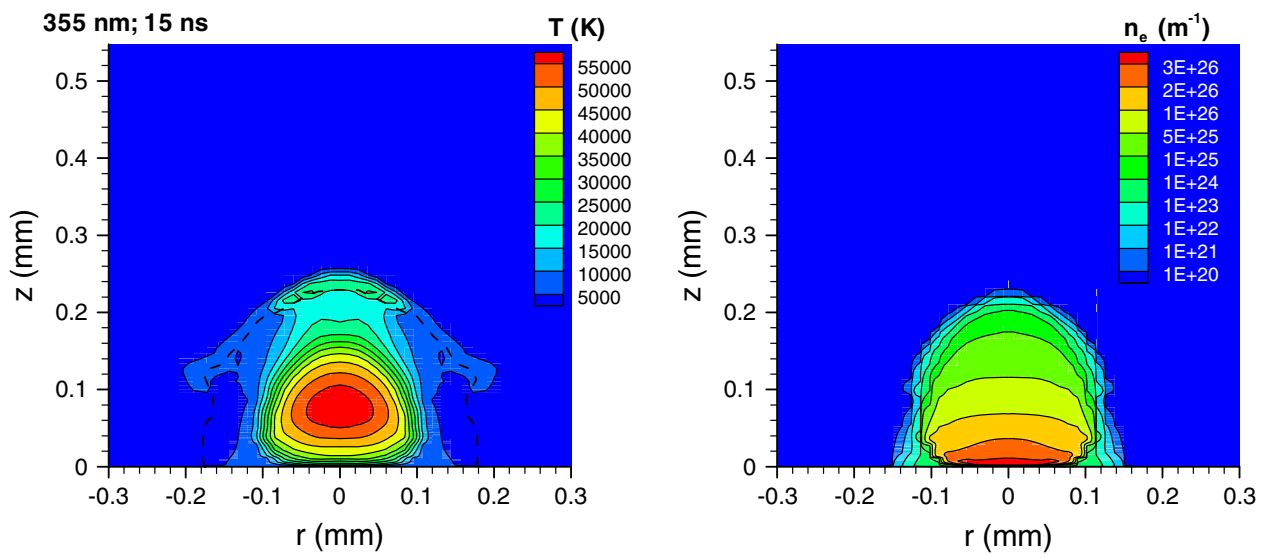
Fig. 5 Distribution of the electron density at $15 \mathrm{~ns}$ from the beginning of the $1,064 \mathrm{~nm}$ laser pulse. Laser fluence $15 \mathrm{~J} \mathrm{~cm}^{-2}$

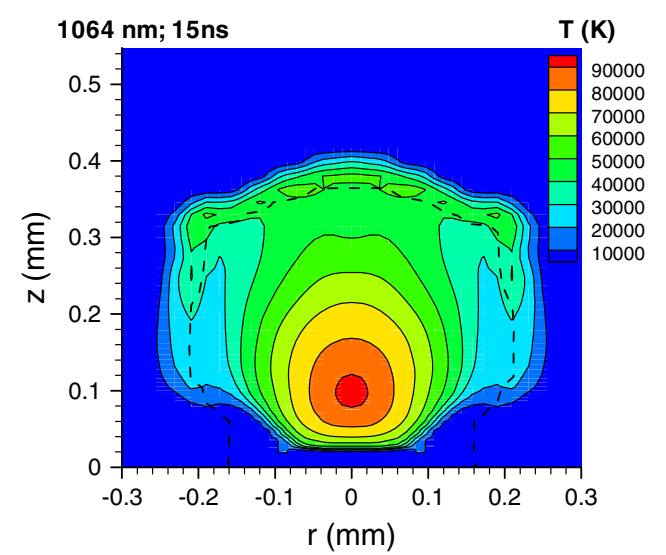

in [26], i.e., at $F \sim 6 \mathrm{~J} \mathrm{~cm}^{-2}$, the ablation depth was significantly lower for $532 \mathrm{~nm}$ than for $1,064 \mathrm{~nm}$ laser wavelength. The explanation given in [26] is that for $532 \mathrm{~nm}$, the penetration depth is lower due to higher absorption coefficient. But on the other hand, our calculations which use similar coefficients give different results. Either the reflectivity or absorption coefficients used in calculations are not correct for the liquid phase of graphite or the optical properties of the surface change during the large number of shots. However, the decrease of the reflectivity from 0.3 to 0.1 [27] corresponds merely to the increase of the absorbed laser fluence by $29 \%$, which does not change the results significantly.

\section{Conclusions}

Similar ablation rate in the case of HOPG and polycrystalline graphite indicates that as a result of laser irradiation, the target looses its crystalline structure and the laser beam interacts with carbon in the liquid phase. The transition from thermal ablation to the phase explosion takes place at lower fluences for shorter laser wavelengths, which means that also in liquid phase, the absorption coefficient of carbon decreases for longer wavelengths. In presented experimental conditions, the phase explosion threshold was at $F \approx 10 \mathrm{~J} \mathrm{~cm}^{-2}$ for $355 \mathrm{~nm}$, at $F \approx 25 \mathrm{~J} \mathrm{~cm}^{-2}$ for $532 \mathrm{~nm}$ and at $F \approx 55 \mathrm{~J} \mathrm{~cm}^{-2}$ for $1,064 \mathrm{~nm}$. The change of the crater shape in the vicinity of the phase explosion threshold was observed. The effect of deep drilling starts in the centre of the laser-irradiated spot because in the case of Gaussian beam, the critical temperature is reached first in the central region of the spot.

The theoretical model predicts a reasonable crater's depth below the threshold. It also gives reasonable values of the ablation rate, although the fact that at low fluences $\left(F \leq 10 \mathrm{~J} \mathrm{~cm}^{-2}\right)$, the ablation rate is higher for the shorter wavelengths, which is not supported by the experiment.
The calculations of plasma radiation flux to the target performed at fluence of $15 \mathrm{~J} \mathrm{~cm}^{-2}$ revealed that at this fluence, the plasma radiation increases the absorbed energy density by 22.5 and $3.8 \%$, in the case of 355 and 1,064 nm radiation, respectively.

Acknowledgments This work was supported by the Polish National Science Centre (Research Project N N501 069138).

Open Access This article is distributed under the terms of the Creative Commons Attribution License which permits any use, distribution, and reproduction in any medium, provided the original author(s) and the source are credited.

\section{References}

1. A. Miotello, R. Kelly, Appl. Phys. Lett. 67, 3535 (1995)

2. J.H. Yoo, S.H. Jeong, R. Greif, R.E. Russo, J. Appl. Phys. 88 , $1638(2000)$

3. N.M. Bulgakova, A. Bulgakov, Appl. Phys. A 73, 199 (2001)

4. N.M. Bulgakova, A.B. Evtushenko, Y.G. Shukhov, S.I. Kudryashov, A.V. Bulgakov, Appl. Surf. Sci. 257, 10876 (2011)

5. C. Porneala, D.A. Willis, Appl. Phys. Lett. 89, 211121 (2006)

6. P. Lorazo, L.J. Lewis, M. Meunier, Phys. Rev. B 73, 134108 (2006)

7. S. Tao, Y. Zhou, B. Wu, Y. Gao, Appl. Surf. Sci. 258, 7766-7773 (2012)

8. P.G. Debenedetti, Metastable Liquids: Concepts and Principles (Princeton University Press, Princeton, 1996)

9. T. Yoshitake, T. Nishiyama, H. Aoki, K. Suizu, K. Takahashi, K. Nagayama, Diam. Rel. Mat. 8, 463 (1999)

10. D. Bolgiaghi, A. Miotello, P. Mosaner, P.M. Ossi, G. Radnoczi, Carbon 43, 2122 (2005)

11. M. Bonelli, A. Miotello, P.M. Ossi, A. Pessi, S. Gialanella, Phys. Rev. B 59, 13513 (1999)

12. J. Hunter, J. Fye, M.F. Jarrold, Science 260, 784 T (1993)

13. A. Puretzky, H. Schittenhelm, X. Fan, M.J. Lance, F.L. Allard Jr, D.B. Geohegan, Phys. Rev. B. 65, 245425 (2002)

14. P. Nikolaev, W. Holmes, E. Sosa, P. Boul, S. Arepalli, L. Yowell, J. Nanosci. Nanotechnol. 10, 3780 (2010)

15. G. Compagnini, M. Sinatra, P. Russo, G.C. Messina, O. Puglisi, S. Scalese, Carbon 50, 2347 (2012)

16. S.Z. Mortazavi, P. Parvin, A. Reyhani, Laser Phys. Lett. 9, 547 (2012)

17. A.T.T. Koh, Y.M. Foong, D.H.C. Chua, Appl. Phys. Lett. 97, $114102(2010)$ 
18. J. Wolowski, P. Gasior, J. Hoffman, M. Kubkowska, M. Rosinski, Z. Szymanski, Radiat. Eff. Defects Solids 165, 434 (2010)

19. R. Windholtz, P.A. Molian, J. Mater. Sci. 32, 4295 (1997)

20. A. Semerok, S.V. Fomichev, F. Brygo, P.Y. Thro, C. Grisolia, J. Nucl. Mater. 420, 198 (2012)

21. V.N. Lednev, S.M. Pershin, E.D. Obraztsova, S.I. Kudryashov, A.F. Bunkin, J. Phys. D Appl. Phys. 46, 052002 (2013)

22. T. Moscicki, J. Hoffman, Z. Szymanski, J. Appl. Phys. 114, 083306 (2013)

23. A. Borghesi, G. Guizzetti, in Handbook of Optical Constants of Solids II, ed. by E.D. Palik (Academic, New York, 1991)
24. D.H. Reitze, H. Ahn, M.C. Downer, Phys. Rev. B 45, 2677 (1992)

25. S.N. Nahar, NORAD-Atomic-Data (Nahar_OSU_Radiative Atomic_Data).http://www.astronomy.ohio-state.edu/ nahar/nahar_ radiativeatomicdata/index.html

26. N.M. Bulgakova, A. Bulgakov, O.L. Bobrenok, Phys. Rev. E 62, $5624(2000)$

27. A.M. Malvezzi, N. Bloembergen, C.Y. Huang, Phys. Rev. Lett. 57, 146 (1986) 